\title{
Antimicrobial resistance of bacteraemia in the emergency department of a German university hospital (2013-2018): potential carbapenem-sparing empiric treatment options in light of the new EUCAST recommendations
}

Kathrin Rothe ${ }^{1,2^{*}}$, Nina Wantia ${ }^{1,2}$, Christoph D. Spinner ${ }^{2,3}$, Jochen Schneider ${ }^{2,3}$, Tobias Lahmer ${ }^{2,3}$, Birgit Waschulzik ${ }^{4}$, Roland M. Schmid ${ }^{2,3}$, Dirk H. Busch ${ }^{1,2}$ and Juri Katchanov ${ }^{2,3}$

\begin{abstract}
Background: This study investigated predominant microorganisms causing community-onset bacteraemia at the medical emergency department (ED) of a tertiary-care university hospital in Germany from 2013 to 2018 and their antimicrobial susceptibility patterns.

Methods: Antimicrobial resistance patterns in patients with positive blood cultures presenting to an internal medicine ED were retrospectively analysed.

Results: Blood cultures were obtained at 5191 of 66,879 ED encounters, with 1013 (19.5\%) positive results, and true positive results at 740 encounters (diagnostic yield, 14.3\%). The most frequently isolated relevant microorganisms were Enterobacterales ( $n=439,59.3 \%)$, Staphylococcus aureus $(n=92,12.4 \%)$, Streptococcus pneumoniae $(n=34$, $4.6 \%)$, Pseudomonas aeruginosa $(n=32,4.3 \%)$, Streptococcus pyogenes $(n=16,2.2 \%)$, Enterococcus faecalis $(n=18$, $2.4 \%)$, and Enterococcus faecium ( $n=12,1.6 \%$ ). Antimicrobial susceptibility testing revealed a high proportion of resistance against ampicillin-sulbactam in Enterobacterales (42.2\%). The rate of methicillin-resistant Staphylococcus aureus was low $(0.4 \%)$.

Piperacillin-tazobactam therapy provided coverage for $83.2 \%$ of all relevant pathogens using conventional breakpoints. Application of the new European Committee on Antimicrobial Susceptibility Testing (EUCAST) recommendations increased the percentage of susceptible isolates to high-dose piperacillin-tazobactam to $92.8 \%$ $(p<0.001)$. Broad-spectrum carbapenems would only cover an additional $4.8 \%$. The addition of vancomycin or linezolid extended coverage by just $1.7 \%$.

(Continued on next page)
\end{abstract}

\footnotetext{
* Correspondence: kathrin.rothe@tum.de

${ }^{1}$ Technical University of Munich, School of Medicine, Institute for Medical

Microbiology, Immunology and Hygiene, Trogerstr. 30, 81675 Munich,

Germany

${ }^{2}$ German Centre for Infection Research (DZIF), Partner Site Munich, Munich,

Germany

Full list of author information is available at the end of the article
}

C The Author(s). 2019 Open Access This article is distributed under the terms of the Creative Commons Attribution 4.0 International License (http://creativecommons.org/licenses/by/4.0/), which permits unrestricted use, distribution, and reproduction in any medium, provided you give appropriate credit to the original author(s) and the source, provide a link to the Creative Commons license, and indicate if changes were made. The Creative Commons Public Domain Dedication waiver (http://creativecommons.org/publicdomain/zero/1.0/) applies to the data made available in this article, unless otherwise stated. 
(Continued from previous page)

Conclusions: Using an ureidopenicillin-beta-lactamase inhibitor combination at the high dose suggested by the new EUCAST recommendations provided nearly 93\% coverage for relevant pathogens in patients with suspected bloodstream infection in our cohort. This might offer a safe option to reduce the empiric use of carbapenems. Our data support the absence of a general need for glycopeptides or oxazolidinones in empiric treatment.

Keywords: Community-onset bacteraemia, Emergency department, Blood cultures, EUCAST, Antimicrobial susceptibility pattern, Treatment regimen

\section{Background}

In the United States, bacteraemia affects approximately 200, 000 patients per year, with 10 per 1000 cases requiring hospital admission [1]. The incidence of community-acquired bloodstream infections is increasing [2]. Previous studies in Denmark and Canada, for example, reported incidence rates around 100 episodes per 100,000 per year [3, 4]. In Europe, there are only a few population-based surveillance systems $[5,6]$. Community-onset bacteraemia is a common problem in patients visiting emergency departments (EDs) [7, 8], and bacteraemia is associated with significant healthcare costs and mortality. The bacteraemia-associated mortality rate has been shown to decrease with early administration of appropriate antimicrobial therapy [9-13]. Thus, ED physicians must regularly initiate empirical antimicrobial therapy before the results of blood cultures (BCs) are available.

$\mathrm{BCs}$ are the gold standard and the most important first-line tool for diagnosing bacteraemia and severe bacterial infections, including sepsis [14-17]. Diagnostic bundles, especially blood culture diagnostics in the ED setting, have been shown to increase numbers of sepsis diagnoses [18], and educational interventions have been shown to improve outcomes in patients with sepsis [19]. Sepsis detection is vital for optimal patient care in the ED setting, as sepsis mortality is reduced when correct antimicrobial therapy is initiated promptly [20-22]. According to national and international sepsis guidelines [23, 24], empiric broad-spectrum antimicrobial therapy with coverage of the most likely pathogens must be initiated immediately in patients with sepsis, including those with bacteraemia $[20,21]$. Additionally, it is an important concept in sepsis care to de-escalate antimicrobial therapy once pathogen identification and resistance testing are completed, or to discontinue empiric antimicrobial therapy when infection can be ruled out [23].

When initiating empiric antibiotic therapy, the potential infectious focus, emergence of multi-resistant pathogens, and economic efficiency must be considered [25]. Common regimens of empiric antimicrobial therapy in the ED are aminopenicillin-beta-lactamase inhibitor combinations (such as ampicillin-sulbactam), ureidopenicillin-betalactamase inhibitor combinations (such as piperacillintazobactam), second and third generation cephalosporins (such as cefuroxime and ceftriaxone), and carbapenems (such as imipenem and meropenem). Hence, knowledge of the expected spectrum of pathogens and antimicrobial resistance is of paramount importance.

The German national 2015 GERMAP report [26] showed that over $10 \%$ of Escherichia coli strains from hospital care in Germany were extended-spectrum beta-lactamase positive, with a piperacillin-tazobactam resistance rate of $6.2 \%$. Hospital strains of Klebsiella oxytoca and Enterobacter cloacae showed piperacillin-tazobactam resistance rates as high as 20.8 and $33.5 \%$, respectively.

However, data on expected pathogens and antimicrobial susceptibility, particularly in community-acquired bacteraemia, are remarkably sparse in Europe [27-32]. Guidelines on antibiotic stewardship recommend adapting guidelines for empiric therapy to local microbiological data and resistance rates to reduce the use of reserve antibiotics. The empiric therapy must be effective, cover the most common pathogens and its prompt initiation is associated with more favourable outcomes [33]. However, to avoid the emergence of antimicrobial resistance, the narrowest spectrum agents and de-escalation strategies are crucial [34]. In addition, in January 2019, the European Committee on Antimicrobial Susceptibility Testing (EUCAST) modified the definitions to categorise susceptibility testing of microorganisms, resulting in a new classification of bacteria as susceptible-standard exposure, susceptible-increased exposure (formerly intermediate), and resistant [35]. This new classification is now related to adequate dosing and exposure at the site of infection. Therefore, ED physicians must be aware of recommended dosing strategies, which have to be addressed at educational visits by the clinical microbiologist or the antibiotic stewardship team.

We conducted this study to expand existing knowledge on expected pathogens and antimicrobial susceptibility in community-acquired bacteraemia in Europe, as well as to explicitly analyse whether the very recent major alterations in EUCAST susceptibility testing classification have implications for empirical antimicrobial therapy.

Thus, we aimed to investigate the predominant microorganisms causing community-onset bacteraemia at the medical ED of a tertiary care university hospital in Germany and their antimicrobial susceptibility patterns, in consideration of the new EUCAST recommendations. 


\section{Methods}

We performed a retrospective analysis of all BCs collected at the internal medicine ED of a university hospital with approximately 1200 beds, located at the centre of Munich, Germany, between 1 October 2013 and 31 September 2018, using the HyBase ${ }^{\bullet}$ analysis system (epiNet AG, Bochum, Germany). The medical ED is responsible for all non-surgical emergencies.

Major indications for ED physicians to suspect infection, obtain BCs [36], and consider antimicrobial therapy include the following: clinically suspected organ infection with accompanying bacteraemia (such as meningitis, cholecystitis, pyelonephritis, necrotising fasciitis, osteomyelitis, severe pneumonia, endocarditis, vascular graft infection, or prosthetic joint infection), clinically suspected sepsis, defined by published sepsis scores, such as the sepsis-related organ failure assessment score, quickSOFA (qSOFA) [22] or SIRS criteria [37], or detected or reported body core temperature $\geq 38.3^{\circ} \mathrm{C}$.

Elevated white blood cell count, C-reactive protein, and procalcitonin, as well as patient's clinical status, were also taken into consideration for obtaining BCs and empiric initiation of antimicrobial therapy. The actual decision to start antibiotic therapy was at the discretion of the physician in charge.

Blood was obtained at the bedside exclusively by physicians according to national practice after skin decontamination using the local antiseptic Octeniderm ${ }^{\oplus}$ (Schülke \& Mayr GmbH, Norderstedt, Germany). Blood was inoculated into aerobic and anaerobic BC media (BACTEC $^{\mathrm{T} \mathrm{M}}$ Plus, Becton Dickinson, Sparks, MD, United States of America) suitable for processing via an automated BC system (BACTEC ${ }^{\mathrm{T}}$ Fluorescent Series, Becton Dickinson). The culture bottles were incubated for 5-7 days according to the manufacturer's recommendations. Immediate Gram stain identification, species identification (Matrix Assisted Laser Desorption Ionization-Time of Flight Mass Spectrometry, Bruker Daltronics, Leipzig, Germany) and automated antimicrobial susceptibility testing (VITEK ${ }^{\oplus}$, bioMerieux, Marcy l'Etoile, France) were performed for all positive cultures. Anaerobic strains were tested using minimal inhibitory concentration (MIC) test strips (Liofilchem Inc., Waltham, MA, United States of America).

The majority of $\mathrm{BC}$ specimens were obtained by peripheral venepuncture and directly from indwelling catheters when catheter-related bacteraemia was suspected. The yield of true bacteraemia was defined as the percentage of episodes with positive $\mathrm{BC}$ results, yielding true positive pathogens (not contaminants such as coagulase-negative staphylococci [CoNS] from a single culture alone) for all episodes during which BCs were obtained. All isolates were categorised as true-positives or contaminants by dichotomous categorisation after critical assessment by at least two investigators, including a clinical microbiologist and a clinical care physician.

Considering the difficulty in determining the clinical significance of CoNS, these isolates were reviewed separately on the basis of the number of positive culture sets, the presence of intravascular devices or indwelling catheters with catheter-related bloodstream infection (CLABSI), the presence of prosthetic heart valves, and patients' characteristics [9]. The isolates were considered clinically significant if two or more bottles out of two or three BC sets yielded the same CoNS [9]. Prosthetic valve endocarditis was extremely scarce in the study population. Hence, the detection of CoNS was usually benign, and often represented contamination. Therefore, we excluded CoNS from considerations for empiric antimicrobial therapy and the term relevant positive $\mathrm{BCs}$ is defined as true positive $\mathrm{BCs}$ excluding true CoNS -bacteraemia.

Additionally, assessment for bacteraemia with viridans group streptococci [38-40] was carried out on the basis of type (e.g., 'abscess-forming' Streptococcus anginosus group versus 'low-virulence' Streptococcus mitis group), focus of infection, and patients' risk factors (such as relevant systemic immunosuppression) to discriminate true positive bacteraemia from contamination. The focus of infection was defined as the clinically most likely site of infection responsible for bacteraemia based on the analysis of clinical signs, microbiological findings, and imaging results $[9,41]$.

EUCAST has very recently modified the definitions for categorisation of susceptibility testing of microorganisms, resulting in a new classification system of bacteria as susceptible-standard exposure, susceptible-increased exposure, and resistant. EUCAST clinical breakpoints 2012-2019 (breakpoint tables version 2.0-version 9.0) for Enterobacterales and Pseudomonas aeruginosa were therefore revised regarding variability in MIC breakpoints for ampicillinsulbactam, piperacillin-tazobactam, imipenem, meropenem, cefuroxime, ceftriaxone, ceftazidime, ciprofloxacin, and moxifloxacin. When the MIC breakpoint values did not at all differ during the entire period, the new EUCAST intermediate definition (susceptible-increased exposure) was hypothetically retrospectively applied, leading to an increase in treatable (susceptible-increased exposure) isolates for ampicillin-sulbactam, piperacillin-tazobactam, meropenem, cefuroxime, ceftriaxone, and ceftazidime.

Statistical analyses were performed using IBM SPSS Statistics V.25.0 (IBM Corp, Armonk, NY). To test the consistency in responses of susceptibility-testing across the two variables 'novel EUCAST classification' and 'conventional breakpoints' we used the McNemar test (two-sided, significance level of 0.05). Exact 95\% confidence intervals were calculated for the percentages of covered pathogens (using the online calculator https:// www.causascientia.org/math_stat/ProportionCI.html). 


\section{Results}

During the study period, 66,879 patient encounters within the ED were recorded. Female and male patients were equally represented in the ED (49.8 and 50.2\%, respectively). The mean age was $54.2( \pm 20.8)$ years.

Presentations through the ambulance and emergency medical services accounted for 35\% of all ED encounters, while walk-in patients accounted for the remaining $65 \%$. BCs were obtained at 5191 encounters (7.8\%); 37,850 encounters resulted in admission to the hospital (56.6\%), with approximately $2.5 \%$ of patients admitted directly to intensive care units.

True bacteraemia was detected in 740 ED cases, resulting in a $\mathrm{BC}$ diagnostic yield of $14.3 \%$ (740 out of 5191). The rate of community-onset true bacteraemia among patients presenting to the ED was $1.1 \%$, and 42 bacteraemia episodes were polymicrobial $(5.6 \%$ of true bacteraemia episodes).

Pathogens considered as contaminants were present in $4.1 \%$ of all positive BCs. Contaminants were the only cause of bacteraemia in 266 episodes (5.1\%) of all ED encounters in which BCs were obtained and $26.3 \%$ of all encounters in which bacteraemia was detected. As the detection of CoNS was usually benign, and often represented contamination without a need for initial empiric antimicrobial therapy, they were excluded from further considerations for schemas regarding empiric antimicrobial therapy.

Consistent with previously published data [28], Enterobacterales were the most commonly isolated pathogens in cases of true bacteraemia $(n=439,59.3 \%)$ followed by Staphylococcus aureus $(n=92,12.4 \%)$. Streptococcus species accounted for $13.5 \%$ of true bacteraemia with Streptococcus pneumoniae $(n=19)$ being the most frequently isolated, followed by Streptococcus pyogenes $(n=16)$. Pseudomonas aeruginosa $(n=32)$ and Enterococcus species $(n=30)$ bacteraemia represented 4.3 and $4.0 \%$ of all true bacteraemia cases, respectively.

Anaerobes accounted only for a small fraction of community-onset bacteraemia cases in our ED cohort: 12 bacteraemia episodes with Bacteroides species (Bacteroides fragilis was detected in 10 episodes) and 12 bacteraemia episodes with Clostridium species (7 of these being Clostridium perfringens) were observed. All except one anaerobic isolate were susceptible to ampicillin-sulbactam: only one detected Bacteroides fragilis isolate was ampicillin-sulbactam-resistant.

After exclusion of CLABSI with CoNS, which do not obligatorily have to be covered by empirical treatment in the ED as described above, bloodstream infections (BSI) with Enterobacterales, Staphylococcus aureus, Streptococcus species, Pseudomonas aeruginosa, and Enterococcus species accounted for more than $95 \%$ of all episodes of relevant positive $\mathrm{BCs}$ in the $\mathrm{ED}$. Table 1 summarises the results of antimicrobial susceptibility testing for those pathogens most commonly isolated in the present study.
The new category 'susceptible-increased exposure' within the novel EUCAST classification broadens the susceptible zone if adequate dosing and exposure at the site of infection are guaranteed. For Enterobacterales and Pseudomonas aeruginosa, hypothetical retrospective application of the new EUCAST definitions resulted in a significant increase in isolates treatable by ampicillin-sulbactam $(p<0.001)$, piperacillin-tazobactam $(p<0.001)$ and cefuroxime $(\mathrm{p}<$ $0.001)$ if dosing recommendations were obeyed. Assuming that all isolates of Enterobacterales $(n=439)$ and Pseudomonas aeruginosa $(n=32)$ in bacteraemia formerly classified as 'intermediate' $(n=37)$ would have been treated with carbapenems and could now be treated with piperacillintazobactam, this results in a hypothetical reduction of carbapenem use of $7.9 \%$ for Enterobacterales and Pseudomonas aeruginosa.

Common regimens of empiric antimicrobial therapy in the ED such as ampicillin-sulbactam, piperacillintazobactam, cephalosporins, and carbapenems were analysed regarding enclosure of the microorganism detected as causing bacteraemia in the study population. Table 2 summarises the percentage of BSI that would be covered by these different empiric antimicrobial regimens in the ED population.

\section{Specific bacteraemia cases}

BSI with Pseudomonas aeruginosa was detected at 32 ED encounters. All affected patients had pre-existing medical conditions and frequently had prior contact with the health care system. Advanced cancer was present in 24 of these patients (75\%) with hematologic malignancy accounting for half of these cases. Neutropenia was present in 12 patients and status post solid organ transplantation in 4 patients with Pseudomonas aeruginosa bacteraemia.

BSI with Enterococcus faecium was rare in patients presenting to the ED; only 12 cases were detected over the 5-year study period. Six patients suffered from acute obstructive cholangitis, two patients from peritoneal dialysis-associated peritonitis, and five patients from advanced cancer.

\section{Discussion}

In ED patients with suspected BSI, antimicrobial therapy has to be initiated before the causative microorganism can be identified and often also before the focus of the infection is unequivocally identified [25]. BC remains the gold standard and first line tool for diagnosing severe bacterial (bloodstream) infections [14, 15]. The detection of relevant microorganisms in the $\mathrm{BC}$ facilitates the search for an infectious focus and allows adjustment to a directed antimicrobial therapy. Only prompt pathogen identification and susceptibility testing allows deescalation of empiric antimicrobial therapy [34] and 
Table 1 Antimicrobial susceptibility data for the main pathogens in BSI in the ED

\begin{tabular}{|c|c|c|c|c|c|c|c|}
\hline $\begin{array}{l}\text { Microorganisms (number of isolates, } \\
\text { percentage of all relevant positive } \\
\mathrm{B}(\mathrm{s}), n=643\end{array}$ & $\begin{array}{l}\text { Staphylococcus } \\
\text { aureus } \\
(n=92,12.4 \%)\end{array}$ & $\begin{array}{l}\text { Streptococcus } \\
\text { pyogenes } \\
(n=16,2.2 \%)\end{array}$ & $\begin{array}{l}\text { Streptococcus } \\
\text { pneumoniae } \\
(n=34,4.6 \%)\end{array}$ & $\begin{array}{l}\text { Enterococcus } \\
\text { faecalis } \\
(n=18 \\
2.4 \%)\end{array}$ & $\begin{array}{l}\text { Enterococcus } \\
\text { faecium } \\
(n=12 \\
1.6 \%)\end{array}$ & $\begin{array}{l}\text { Enterobacterales } \\
(n=439 \\
59.3 \%)\end{array}$ & $\begin{array}{l}\text { Pseudomonas } \\
\text { aeruginosa } \\
(n=32,4.3 \%)\end{array}$ \\
\hline Penicillin & N/A & $16(100 \%)$ & $33(97.1 \%)$ & - & - & - & - \\
\hline Ampicillin & N/A & $16(100 \%)$ & $33(97.1 \%)$ & $18(100 \%)$ & $3(25 \%)$ & $155(35.3 \%)$ & - \\
\hline Flucloxacillin & $89(96.7 \%)$ & $\mathrm{N} / \mathrm{A}$ & - & - & - & - & - \\
\hline Ampicillin-sulbactam & $89(96.7 \%)$ & $16(100 \%)$ & $33(97.1 \%)$ & $18(100 \%)$ & $3(25 \%)$ & $\begin{array}{l}230(52.4 \%) \\
253^{*}(57.8 \%)\end{array}$ & - \\
\hline Piperacillin-tazobactam & $89(96.7 \%)$ & $16(100 \%)$ & $33(97.1 \%)$ & $18(100 \%)$ & $3(25 \%)$ & $\begin{array}{l}376(85.6 \%) \\
411^{*}(93.8 \%)\end{array}$ & $\begin{array}{l}25(78.1 \%) \\
27(84.4 \%)\end{array}$ \\
\hline Imipenem & $89(96.7 \%)$ & $16(100 \%)$ & $34(100 \%)$ & $18(100 \%)$ & $3(25 \%)$ & $426(97.0 \%)$ & $26(81.3 \%)$ \\
\hline Meropenem & $89(96.7 \%)$ & $16(100 \%)$ & $34(100 \%)$ & - & - & $\begin{array}{l}439(100 \%) \\
439^{*}(100 \%)\end{array}$ & $\begin{array}{l}26(81.3 \%) \\
29^{*}(90.6 \%)\end{array}$ \\
\hline 2nd gen Cephalosporin (cefuroxime) & $89(96.7 \%)$ & $16(100 \%)$ & $33(97.1 \%)$ & - & - & $\begin{array}{l}327(74.5 \%) \\
347^{*}(79.0 \%)\end{array}$ & - \\
\hline $\begin{array}{l}\text { 3rd gen Cephalosporin } \\
\text { (ceftriaxone) }\end{array}$ & $89(96.7 \%)$ & $16(100 \%)$ & $34(100 \%)$ & - & - & $\begin{array}{l}388(88.4 \%) \\
389^{*}(88.6 \%)\end{array}$ & - \\
\hline 4th gen Cephalosporin (ceftazidime) & - & - & - & - & - & $\begin{array}{l}385(87.7 \%) \\
412^{*}(93.8 \%)\end{array}$ & $\begin{array}{l}28(87.5 \%) \\
29^{*}(90.6 \%)\end{array}$ \\
\hline Ciprofloxacin & $86(93.5 \%)$ & - & - & N/A & N/A & $343(78.1 \%)$ & $26(81.3 \%)$ \\
\hline Levofloxacin & $86(93.5 \%)$ & $15(93.8 \%)$ & $34(100 \%)$ & N/A & N/A & N/A & N/A \\
\hline Moxifloxacin & $86(93.5 \%)$ & N/A & N/A & - & - & $322(73.3 \%)$ & - \\
\hline Vancomycin & $92(100 \%)$ & $16(100 \%)$ & $34(100 \%)$ & $18(100 \%)$ & $12(100 \%)$ & - & - \\
\hline Linezolid & $92(100 \%)$ & $16(100 \%)$ & $34(100 \%)$ & $18(100 \%)$ & $12(100 \%)$ & - & - \\
\hline
\end{tabular}

For Enterobacterales and Pseudomonas aeruginosa, the percentage of susceptible isolates is presented according to the interpretation of breakpoints at the time of testing. According to the novel EUCAST definition of the intermediate category, therapeutic success is likely if exposure is increased. This was hypothetically retrospectively applied, leading to an increase in susceptible isolates if dosing recommendations were obeyed (marked with *). "-" indicates that the species is a poor target for therapy with the agent; isolates may be considered as resistant. N/A indicates not available/applicable; relevant positive BCs are defined as true positive BCs excluding true CoNS -bacteraemia.

widens existing knowledge of the bacterial spectrum and antimicrobial resistance in BSI; therefore, BC represents a major pillar of antibiotic stewardship [42]. It is of paramount importance for ED physicians to be aware of the expected spectrum of pathogens in bacteraemia and antimicrobial susceptibilities. In accordance with previous studies, our data show that Enterobacterales, Staphylococcus aureus, Streptococcus species, and Pseudomonas aeruginosa account for the majority of clinically relevant BSI in the ED [9, 43-45].

In our study population, empirical therapy with ureidopenicillin-tazobactam covered $83.2 \%$ of all relevant pathogens. However, this proportion increased to $92.8 \%$ after including the isolates that were hypothetically retrospectively classified under the category of susceptibleincreased exposure according to the novel EUCAST definitions. Applying the novel EUCAST breakpoints and using the high-dose antimicrobial regimens would therefore provide an option of broad microbiological efficacy in the vast majority of patients presenting with communityonset bacteraemia, while reducing carbapenem use. However, in this potential antibiotic stewardship intervention approach, awareness of high-dose recommendations is crucial. The education of physicians, nurses, and other staff might be essential for the success of such a strategy. Using a broad spectrum carbapenem would only cover an additional $4.8 \%$ of relevant pathogens (28 isolates of Enterobacterales and three isolates of Pseudomonas aeruginosa in the study population). In the study population, of 35 isolates of Enterobacterales that were retrospectively hypothetically classified under the category of susceptible-increased exposure, 19 (54.3\%) tested positive for extended-spectrum beta-lactamase (ESBL).

Treatment of community-onset BSI due to ESBLproducing Enterobacterales with an ureidopenicillinbeta-lactamase inhibitor combination is still a matter of debate and conflicting results have been reported. It has been shown that piperacillin-tazobactam is not inferior to carbapenems for treatment of extended-spectrum beta-lactamase (ESBL)-producing Enterobacterales especially in low-to moderate-severity infections and when adequate dosing regimens ( $4.5 \mathrm{~g}$ every $6 \mathrm{~h}$ or $4.5 \mathrm{~g}$ every $8 \mathrm{~h}$ as prolonged infusion) are used [46-50]. Tamma et al. [51] reported that carbapenem therapy is associated with improved survival compared to piperacillintazobactam in patients with ESBL-bacteraemia. This 


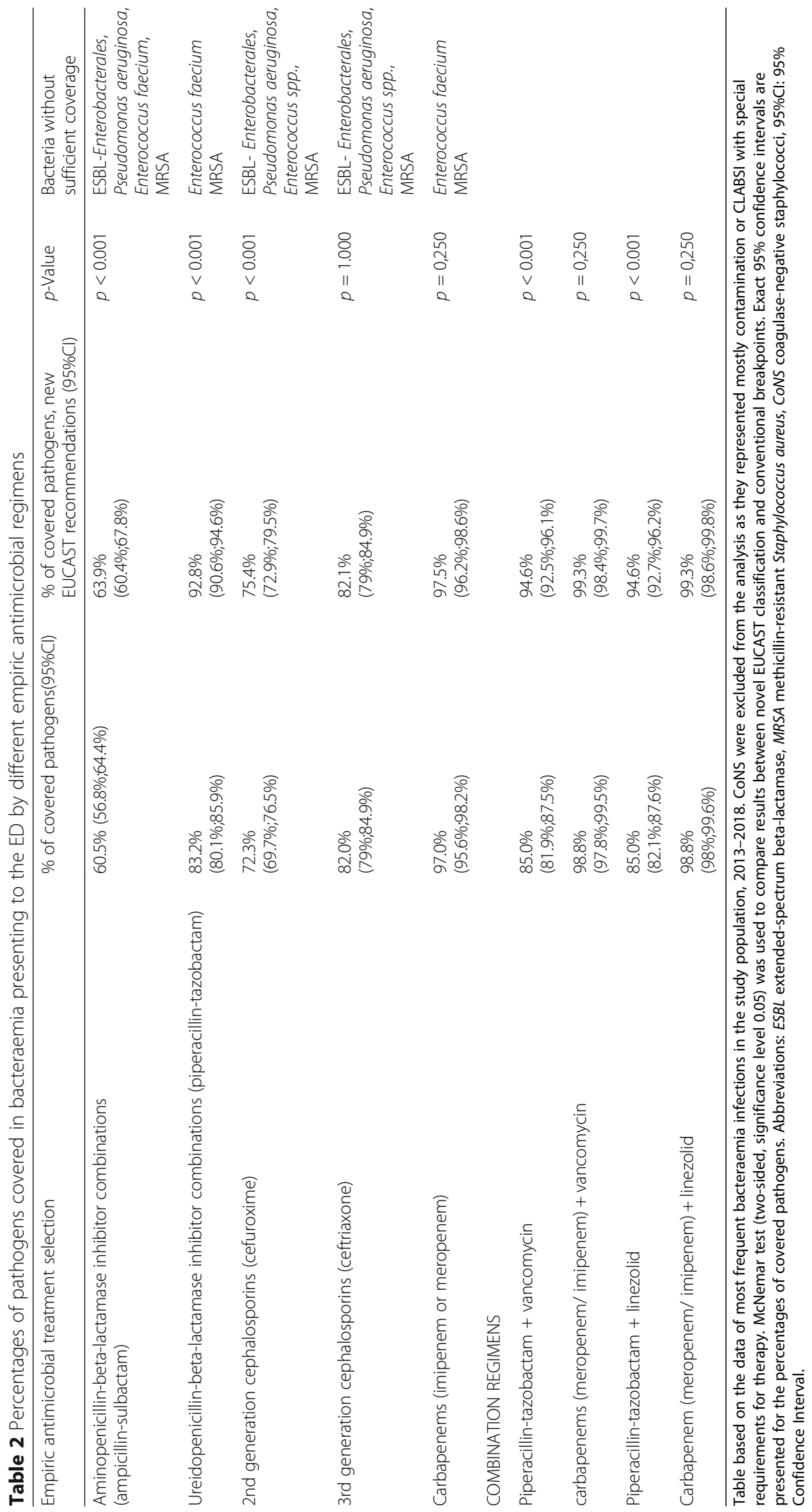


study used piperacillin-tazobactam $3.375 \mathrm{~g}$ every $6 \mathrm{~h}$ in the majority of patients. Notably, when high- dose piperacillin-tazobactam (defined as $4.5 \mathrm{~g}$ every $6 \mathrm{~h}$ ) was administered to a subset of patients in this study, no difference in clinical outcome compared to carbapenem administration was observed [51]. The lack of uniform treatment regimens regarding piperacillin-tazobactamdosing has to be taken into consideration when comparing outcomes of different studies on this topic.

Of note, a study conducted by Harris et al. [49], showing non-inferior 30-day mortality of piperacillin-tazobactamtherapy in ESBL-bacteraemia, explicitly administered piperacillin-tazobactam $4.5 \mathrm{~g}$ every $6 \mathrm{~h}$. In contrast to other studies, in this case susceptibility testing was performed according to EUCAST standards. Remarkably the EUCAST breakpoint for susceptibility testing for piperacillin-tazobactam (susceptible standard exposure: MIC $\leq 8 \mathrm{mg} / \mathrm{L}$ ) is set lower than the Clinical and Laboratory Standards Institute (CLSI) breakpoint (susceptible: $\mathrm{MIC} \leq 16 \mathrm{mg} / \mathrm{L}$ ) [49]. The inconsistent breakpoints of EUCAST and CLSI can result in different interpretation of susceptibility testing, impeding comparability of studies even more. Therefore, additional research is needed to provide data on the question if piperacillin-tazobactam is a safe therapeutic option for critical ill patients with severe infections with ESBL-producing Enterobacterales, especially considering the source of infection, dosing regimens, results of susceptibility testing and MICs [50].

It has been shown, that community onset-bacteraemia with ESBL-producing Escherichia coli, Klebsiella species and Proteus mirabilis is associated with worse clinical outcome compared to non ESBL-producing isolates, but a propensity-matched analysis did not show a significant difference between appropriateness of empirical antibiotic therapy between the groups [52]. Remarkably in the busy ED setting, primarily identification of ESBLbacteraemia by adequate blood culture diagnostic is vital, thus enabling subsequent adjustment of antimicrobial therapy in this population at risk. However, in clinically stable ED-patients in absence of septic condition and low prevalence of ESBL-producers, it seems reasonable to start with empiric antimicrobial therapy according to local resistance rates [53]. Definite antimicrobial therapy should then be adopted to results of pathogen identification, resistance testing and clinical course.

In conclusion, strategies to reduce the use of carbapenems are warranted and safe carbapenem-sparing treatment options with ureidopenicillin-beta-lactamase inhibitor combinations might be one possible approach. Therefore, we propose the option of adequate high-dose ureidopenicillin-betalactamase inhibitor combination therapy for communityonset bacteraemia with the intent to reduce carbapenem overtreatment especially in low-to moderate-severity infections. Nevertheless, awareness of risk factors that accompany the need for distinct antimicrobial coverage and specific therapeutic considerations necessary in critically ill patients (who require intensive care) must be raised.

One particular target population for broad spectrum antibiotic prescription is patients with sepsis. Many patients with bacteraemia fulfil sepsis criteria [54-57], but only a fraction of patients with sepsis have detectable bacteraemia [58-61]. Our study investigated the ED setting of a German tertiary care hospital. The ED physician must recognise suspected sepsis in a timely manner and promptly begin the initial work-up, including obtaining blood cultures and administering broad spectrum antibiotics. The patient's clinical condition is the determining factor for deciding the necessity of initiating antimicrobial therapy and selecting suitable antimicrobial agents. The urgent need to immediately initiate the correct antibiotic in patients with sepsis creates the need for broadspectrum agents [62]. Our study shows that piperacillintazobactam monotherapy at the high dose suggested in the new EUCAST recommendations covers nearly $93 \%$ of bacteraemia cases in our cohort. This offers a safe option for suspected BSI in the ED and reduces empiric use of carbapenems in line with antibiotic stewardship principles. In particular, in clinically stable patients with suspected BSI who require immediate initiation of empiric broadspectrum antibiotics, it seems reasonable to start with a narrower spectrum therapy according to local resistance rates and withhold reserve antibiotics when clinical monitoring for deterioration is provided.

Caution is warranted in the care of patients at risk of multi-resistant pathogens, such as patients with immunosuppression or haematological malignancy. General risk factors for multi-resistant bacteria include previous colonisation, previous hospitalisation, previous antibiotic therapy, patients in long-term-care facilities requiring chronic care or with chronic wounds, and patients with permanent catheters [63-65]. Adding vancomycin or linezolid widened coverage by only an additional $1.7 \%$ in the present study and should therefore be restricted, especially to patients with clinical suspicion for CLABSI or BSI due to methicillin-resistant Staphylococcus aureus. It is not generally needed in routine empiric therapy. In the study population, the rate of methicillin-resistant Staphylococcus aureus (MRSA) in community-acquired BSI was extremely low. Generally, the percentage of invasive Staphylococcus aureus isolates with resistance to methicillin in Germany is decreasing, with a rate of $9.1 \%$ in 2017 [66]. Surveillance systems in Germany show a decline in incidence of MRSA infections to $4.8 / 100,000$ in 2014 with regional differences; incidences were as low as 2.0/100,000 in 2014 in southern Germany [67]. We therefore believe that, in absence of known previous risk factors, it is possible to withhold vancomycin or linezolid from most patients with suspected BSI in our ED setting. 
The present study supports the fact that in patients with suspected BSI the use of reserve antibiotics such as carbapenems, vancomycin and oxazolidinones is only essential in very few cases $(6.5 \%)$ with dedicated risk factors within special populations. Prina and colleagues proposed the acronym 'PES' ('Pseudomonas aeruginosa, Enterobacteriaceae extended-spectrum beta-lactamase-positive and methicillinresistant Staphylococcus aureus') to identify pathogens that require different treatment regimes in community-acquired pneumonia [68]. For BSI in the ED, E. faecium should be considered as a microorganism usually requiring special antimicrobial treatment. However, the number of patients with community-onset E. faecium bacteraemia was very low, and half of the episodes presented as acute cholangitis, where source control was the main pillar of therapy. Moreover, MRSA- bacteraemia was extremely rare, with only three cases observed in the study period. Nevertheless, awareness of the risk factors that accompany the need for distinct antimicrobial coverage must be raised and using an acronym might be one possible method to address this issue. Additional research is needed to provide guidance for the treatment of critically ill patients with septicaemia that require intensive care, with the aim of increasing coverage of empirical antibiotic therapy to $100 \%$.

\section{Limitations}

This study has several limitations. It is a single-centre study based on a retrospective analysis of laboratory data without correlation with clinical outcome data. However, the heterogeneous population of a city-based ED with a large catchment area may allow generalisation to other EDs in Germany and Europe. Another limitation of the present study is that only bacteraemia was considered. The sensitivity of $\mathrm{BC}$ is limited; moreover, some pathogens accounting for acute infections in the ED, such as Legionella pneumophila or fastidious intracellular pathogens, cannot be isolated using $\mathrm{BC}$ techniques. These pathogens must be taken into consideration and clinical indicators must be carefully observed. It is also important to differentiate between the clinical condition of sepsis and the microbiological finding of bacteraemia. Bacteraemia might occur during sepsis, but also affects clinically stable patients without sepsis. Although bacteraemia might strongly correlate with suspected sepsis, the findings of the present study cannot lead to a clinical recommendation for sepsis patients in general, as this study was focused on ED encounters with suspected BSI and antimicrobial resistance of bacteraemia; no clinical data on sepsis diagnosis, antimicrobial treatment, or therapeutic outcome was available.

\section{Conclusions}

$\mathrm{BCs}$ remain the gold standard for diagnosing severe bacterial infections $[14,15]$, representing a major pillar of infection diagnosis and a crucial part of antibiotic stewardship [42]. Our study indicates that, at a tertiary care centre in Germany, empiric therapy with a high-dose ureidopenicillin-beta-lactamase inhibitor combination, in accordance with the novel EUCAST recommendations, might be an appropriate antibiotic stewardship intervention strategy for reducing carbapenem use in patients with suspected community-onset bacteraemia. In our cohort, more than $90 \%$ of detected relevant microorganisms in BSI were successfully covered with this antibiotic regimen. Still, caution is warranted in critically ill patients who require intensive care, patients with risk factors for infections with multi-resistant pathogens [63-65], and/or patients with vascular-device-related BSI, as our data does not address these populations. Here, specific therapeutic considerations are necessary. Moreover, the therapeutic role of high-dose ureidopenicillin-beta-lactamase inhibitor combinations in infections due to extended-spectrum beta-lactamase Enterobacterales must be further clarified in clinical studies. Incorporating the new EUCAST definitions in antibiotic stewardship programmes could enhance carbapenem withholding and restrict the use of reserve antibiotics to fight emerging resistance.

\begin{abstract}
Abbreviations
95\%Cl: 95\% Confidence Interval; BC: Blood culture; BSI: Bloodstream infection; CLABSI: Catheter-related bloodstream infection; CoNS: Coagulase-negative staphylococci; ED: Emergency department; ESBL: Extended-Spectrum BetaLactamase; EUCAST: European Committee on Antimicrobial Susceptibility Testing; MRSA: Methicillin-resistant Staphylococcus aureus
\end{abstract}

\section{Acknowledgements}

Not Applicable.

\section{Authors' contributions}

KR and JK conceived the study, analysed the data, and interpreted the results. CDS, NW, JS made contributions to design of the study and acquisition and interpretation of the data. KR and JK wrote the manuscript BW performed statistical calculations. TL, CDS, RMS, DHB revised it critically for important intellectual content. All authors agree with the article submission. All authors read and approved the final manuscript.

Funding

Not applicable. No funding was obtained for this study.

Availability of data and materials

All relevant data have been made available in the article. Raw data can be requested from the corresponding author.

Ethics approval and consent to participate

The Ethics Committee of the Technical University of Munich approved the protocol for this retrospective study and waived the need to obtain consent for the collection, analysis, and publication of the retrospectively obtained and anonymised data (approval No. 539/185).

Consent for publication

Not applicable.

Competing interests

The authors declare no competing interests.

Author details

${ }^{1}$ Technical University of Munich, School of Medicine, Institute for Medical Microbiology, Immunology and Hygiene, Trogerstr. 30, 81675 Munich, 
Germany. ${ }^{2}$ German Centre for Infection Research (DZIF), Partner Site Munich, Munich, Germany. ${ }^{3}$ Department of Medicine II, Technical University of Munich, School of Medicine, University Hospital rechts der Isar, Munich, Germany. ${ }^{4}$ Institute of Medical Informatics, Statistics, and Epidemiology, Technical University of Munich, Munich, Germany.

Received: 17 June 2019 Accepted: 22 December 2019

Published online: 30 December 2019

\section{References}

1. Long B, Koyfman A. Best clinical practice: blood culture utility in the emergency department. J Emerg Med. 2016;51(5):529-39.

2. Christaki E, Giamarellos-Bourboulis EJ. The complex pathogenesis of bacteremia: from antimicrobial clearance mechanisms to the genetic background of the host. Virulence. 2014;5(1):57-65.

3. Sogaard M, Norgaard M, Dethlefsen C, Schonheyder HC. Temporal changes in the incidence and 30-day mortality associated with bacteremia in hospitalized patients from 1992 through 2006: a population-based cohort study. Clin Infect Dis. 2011;52(1):61-9.

4. Laupland KB, Kibsey PC, Gregson DB, Galbraith JC. Population-based laboratory assessment of the burden of community-onset bloodstream infection in Victoria, Canada. Epidemiol Infect. 2013;141(1):174-80.

5. Schmitz RP, Rissner F, Brunkhorst FM. The Thuringian registry for bloodstream infections, antibiotic resistance and the practice of blood culture sampling--AlertsNet. Int J Antimicrob Agents. 2015;46(Suppl 1):S5-9.

6. Karch A, Schmitz RP, Rissner F, Castell S, Topel S, Jakob M, Brunkhorst FM, Mikolajczyk RT. Bloodstream infections, antibiotic resistance and the practice of blood culture sampling in Germany: study design of a Thuringia-wide prospective population-based study (AlertsNet). BMJ Open. 2015;5(12):e009095.

7. Shapiro NI, Wolfe RE, Wright SB, Moore R, Bates DW. Who needs a blood culture? A prospectively derived and validated prediction rule. J Emerg Med. 2008;35(3):255-64.

8. Villalon N, Farzan N, Freeman K. Rate of bacteremia in the hemodialysis patient presenting to the emergency department with fever: a retrospective chart review. Int J Emerg Med. 2018;11(1):29.

9. Weinstein MP, Towns ML, Quartey SM, Mirrett S, Reimer LG, Parmigiani G, Reller LB. The clinical significance of positive blood cultures in the 1990s: a prospective comprehensive evaluation of the microbiology, epidemiology, and outcome of bacteremia and fungemia in adults. Clin Infect Dis : an official publication of the Infectious Diseases Society of America. 1997;24(4):584-602.

10. Pien BC, Sundaram P, Raoof N, Costa SF, Mirrett S, Woods CW, Reller LB, Weinstein MP. The clinical and prognostic importance of positive blood cultures in adults. Am J Med. 2010;123(9):819-28.

11. Salomao R, Castelo Filho A, Pignatari AC, Wey SB. Nosocomial and community acquired bacteremia: variables associated with outcomes. Rev Paul Med. 1993;111(6):456-61.

12. Chiu CW, Li MC, Ko WC, Li CW, Chen PL, Chang CM, Lee NY, Lee CC. Clinical impact of gram-negative nonfermenters on adults with community-onset bacteremia in the emergency department. J Microbiol Immunol Infect = Wei mian yu gan ran za zhi. 2015;48(1):92-100.

13. Chen HC, Lin WL, Lin CC, Hsieh WH, Hsieh CH, Wu MH, Wu JY, Lee CC. Outcome of inadequate empirical antibiotic therapy in emergency department patients with community-onset bloodstream infections. J Antimicrob Chemother. 2013;68(4):947-53.

14. Bennett IL Jr, Beeson PB. Bacteremia: a consideration of some experimental and clinical aspects. Yale J Biol Med. 1954;26(4):241-62.

15. Dubourg G, Raoult D, Fenollar F. Emerging methodologies for pathogen identification in bloodstream infections: an update. Expert Rev Mol Diagn. 2019;19(2):161-173.

16. Lamy B, Dargere S, Arendrup MC, Parienti JJ, Tattevin P. How to optimize the use of blood cultures for the diagnosis of bloodstream infections? A State-of-the Art. Front Microbiol. 2016;7:697.

17. Garcia RA, Spitzer ED, Beaudry J, Beck C, Diblasi R, Gilleeny-Blabac M, Haugaard C, Heuschneider S, Kranz BP, McLean K, et al. Multidisciplinary team review of best practices for collection and handling of blood cultures to determine effective interventions for increasing the yield of true-positive bacteremias, reducing contamination, and eliminating false-positive central line-associated bloodstream infections. Am J Infect Control. 2015;43(11):1222-37.

18. Whippy A, Skeath M, Crawford B, Adams C, Marelich G, Alamshahi M, Borbon J. Kaiser Permanente's performance improvement system, part 3: multisite improvements in care for patients with sepsis. Jt Comm J Qual Patient Saf. 2011;37(11):483-93.

19. Ferrer R, Martinez ML, Goma G, Suarez D, Alvarez-Rocha L, de la Torre MV, Gonzalez G, Zaragoza R, Borges M, Blanco J, et al. Improved empirical antibiotic treatment of sepsis after an educational intervention: the ABISSEdusepsis study. Crit Care. 2018;22(1):167.

20. Bochud PY, Bonten M, Marchetti O, Calandra T. Antimicrobial therapy for patients with severe sepsis and septic shock: an evidence-based review. Crit Care Med. 2004;32(11 Suppl):S495-512.

21. Siddiqui S, Razzak J. Early versus late pre-intensive care unit admission broad spectrum antibiotics for severe sepsis in adults. Cochrane Database Syst Rev. 2010;(10):CD007081. https://doi.org/10.1002/14651858.CD007081.pub2.

22. Singer M, Deutschman CS, Seymour CW, Shankar-Hari M, Annane D, Bauer M, Bellomo R, Bernard GR, Chiche JD, Coopersmith CM, et al. The third international consensus definitions for Sepsis and septic shock (Sepsis-3). Jama. 2016;315(8):801-10.

23. Levy MM, Evans LE, Rhodes A. The surviving Sepsis campaign bundle: 2018 update. Crit Care Med. 2018;46(6):997-1000.

24. Reinhart K, Brunkhorst F, Bone $\mathrm{H}$, Gerlach $\mathrm{H}$, Grundling M, Kreymann G, Kujath P, Marggraf G, Mayer K, Meier-Hellmann A, et al. Diagnosis and therapy of sepsis: guidelines of the German Sepsis Society Inc. and the German Interdisciplinary Society for Intensive and Emergency Medicine. Der Anaesthesist. 2006;55(Suppl 1):43-56.

25. Leibovici L, Shraga I, Drucker M, Konigsberger H, Samra Z, Pitlik SD. The benefit of appropriate empirical antibiotic treatment in patients with bloodstream infection. J Intern Med. 1998;244(5):379-86.

26. Federal Office of Consumer Protection and Food Safety, Paul-EhrlichGesellschaft für Chemotherapie e.V:: GERMAP 2015 - Report on the consumption of antimicrobials and the spread of antimicrobial resistance in human and veterinary medicine in Germany. Antiinfectives Intelligence, Rheinbach 2016. (https://www.p-e-g.org/files/content/Ueber\%20uns/ GERMAP/GERMAP-2015english.pdf).

27. Sirijatuphat $R$, Sripanidkulchai $K$, Boonyasiri A, Rattanaumpawan $P$, Supapueng O, Kiratisin P, Thamlikitkul V. Implementation of global. antimicrobial resistance surveillance system (GLASS) in patients with bacteremia. PLoS One. 2018;13(1):e0190132.

28. Courjon J, Demonchy E, Degand N, Risso K, Ruimy R, Roger PM. Patients with community-acquired bacteremia of unknown origin: clinical characteristics and usefulness of microbiological results for therapeutic issues: a single-center cohort study. Ann Clin Microbiol Antimicrob. 2017;16(1):40.

29. Najmi A, Karimi F, Kunhikatta V, Varma M, Nair S. Resistance trend, antibiotic utilization and mortality in patients with E. coli Bacteraemia. Open Access Maced J Med Sci. 2019;7(7):1119-23.

30. Lin WP, Huang YS, Wang JT, Chen YC, Chang SC. Prevalence of and risk factor for community-onset third-generation cephalosporin-resistant Escherichia coli bacteremia at a medical center in Taiwan. BMC Infect Dis. 2019;19(1):245.

31. Chen X, Zou Q, Zhang W, Wang R, Yu F, Chen Y. Clinical features and microbiological characteristics of hospital- and community-onset Escherichia coli bloodstream infection. J Med Microbiol. 2019;68(2):178-87.

32. Lee S, Han SW, Kim KW, Song DY, Kwon KT. Third-generation cephalosporin resistance of community-onset Escherichia coli and Klebsiella pneumoniae bacteremia in a secondary hospital. Korean J Intern Med. 2014;29(1):49-56.

33. Lambregts MMC, Hendriks BJC, Visser LG, Bernards ST, de Boer MGJ. Using local clinical and microbiological data to develop an institution specific carbapenem-sparing strategy in sepsis: a nested case-control study. Antimicrob Resist Infect Control. 2019;8:19.

34. de With K, Allerberger F, Amann S, Apfalter P, Brodt HR, Eckmanns T, Fellhauer M, Geiss HK, Janata O, Krause R, et al. Strategies to enhance rational use of antibiotics in hospital: a guideline by the German Society for Infectious Diseases. Infection. 2016;44(3):395-439.

35. The European Committee on Antimicrobial Susceptibility Testing. Breakpoint tables for interpretation of MICs and zone diameters. Version 9.0 hweo.

36. Rothe K, Spinner CD, Ott A, Querbach C, Dommasch M, Aldrich C, Gebhardt F, Schneider J, Schmid RM, Busch DH, et al. Strategies for increasing diagnostic yield of community-onset bacteraemia within the emergency department: a retrospective study. PLoS One. 2019;14(9):e0222545.

37. Marik PE, Taeb AM. SIRS, qSOFA and new sepsis definition. J Thorac Dis. 2017;9(4):943-5.

38. Kobo O, Nikola S, Geffen Y, Paul M. The pyogenic potential of the different Streptococcus anginosus group bacterial species: retrospective cohort study. Epidemiol Infect. 2017;145(14):3065-9. 
39. Shenep JL. Viridans-group streptococcal infections in immunocompromised hosts. Int J Antimicrob Agents. 2000;14(2):129-35.

40. Tunkel AR, Sepkowitz KA. Infections caused by viridans streptococci in patients with neutropenia. Clin Infect Dis : an official publication of the Infectious Diseases Society of America. 2002;34(11):1524-9.

41. Smit J, Rieg SR, Wendel AF, Kern WW, Seifert H, Schonheyder HC, Kaasch AJ. Onset of symptoms, diagnostic confirmation, and occurrence of multiple infective foci in patients with Staphylococcus aureus bloodstream infection: a look into the order of events and potential clinical implications. Infection. 2018; 46(5):651-658. https://doi.org/10.1007/s15010-018-1165-x. Epub 2018 Jun 14.

42. Mathieu C, Pastene B, Cassir N, Martin-Loeches I, Leone M. Efficacy and safety of antimicrobial de-escalation as a clinical strategy. Expert Rev AntiInfect Ther. 2018.

43. Kao CH, Kuo YC, Chen CC, Chang YT, Chen YS, Wann SR, Liu YC. Isolated pathogens and clinical outcomes of adult bacteremia in the emergency department: a retrospective study in a tertiary Referral Center. J Microbiol Immunol Infect = Wei mian yu gan ran za zhi. 2011;44(3):215-21.

44. Stalnikowicz R, Block C. The yield of blood cultures in a department of emergency medicine. Eur J Emerg Med : official journal of the European Society for Emergency Medicine. 2001;8(2):93-7.

45. Boyles TH, Davis K, Crede T, Malan J, Mendelson M, Lesosky M. Blood cultures taken from patients attending emergency departments in South Africa are an important antibiotic stewardship tool, which directly influences patient management. BMC Infect Dis. 2015;15:410.

46. Gutierrez-Gutierrez B, Perez-Galera S, Salamanca E, de Cueto M, Calbo E, Almirante B, Viale P, Oliver A, Pintado V, Gasch O, et al. A multinational, preregistered cohort study of beta-lactam/beta-lactamase inhibitor combinations for treatment of bloodstream infections due to extendedSpectrum-beta-lactamase-producing Enterobacteriaceae. Antimicrob Agents Chemother. 2016;60(7):4159-69.

47. Ng TM, Khong WX, Harris PN, De PP, Chow A, Tambyah PA, Lye DC. Empiric Piperacillin-Tazobactam versus Carbapenems in the treatment of Bacteraemia due to extended-Spectrum Beta-lactamase-producing Enterobacteriaceae. PLoS One. 2016;11(4):e0153696.

48. Rodriguez-Bano J, Navarro MD, Retamar P, Picon E, Pascual A. beta-lactam/ beta-lactam inhibitor combinations for the treatment of bacteremia due to extended-spectrum beta-lactamase-producing Escherichia coli: a post hoc analysis of prospective cohorts. Clin Infect Dis : an official publication of the Infectious Diseases Society of America. 2012;54(2):167-74.

49. Harris PNA, Tambyah PA, Lye DC, Mo Y, Lee TH, Yilmaz M, Alenazi TH, Arabi Y, Falcone M, Bassetti M, et al. Effect of Piperacillin-Tazobactam vs Meropenem on 30-day mortality for patients With E coli or Klebsiella pneumoniae bloodstream infection and ceftriaxone resistance: a randomized clinical trial. Jama. 2018;320(10):984-94.

50. Tamma PD, Rodriguez-Bano J. The use of Noncarbapenem beta-lactams for the treatment of extended-Spectrum beta-lactamase infections. Clin Infect Dis : an official publication of the Infectious Diseases Society of America. 2017;64(7):972-80.

51. Tamma PD, Han JH, Rock C, Harris AD, Lautenbach E, Hsu AJ, Avdic E, Cosgrove SE. Carbapenem therapy is associated with improved survival compared with piperacillin-tazobactam for patients with extendedspectrum beta-lactamase bacteremia. Clin Infect Dis : an official publication of the Infectious Diseases Society of America. 2015;60(9):1319-25.

52. Lee CC, Lee CH, Hong MY, Hsieh CC, Tang HJ, Ko WC. Propensity-matched analysis of the impact of extended-spectrum beta-lactamase production on adults with community-onset Escherichia coli, Klebsiella species, and Proteus mirabilis bacteremia. J Microbiol Immunol Infect = Wei mian yu gan ran za zhi. 2018;51(4):519-26.

53. Lee $\mathrm{CH}$, Hsieh CC, Hong MY, Hung YP, Ko WC, Lee CC. Comparing the therapeutic efficacies of third-generation cephalosporins and broaderspectrum beta-lactams as appropriate empirical therapy in adults with community-onset monomicrobial Enterobacteriaceae bacteraemia: a propensity score matched analysis. Int J Antimicrob Agents. 2017;49(5):617-23.

54. Madsen KM, Schonheyder HC, Kristensen B, Nielsen GL, Sorensen HT. Can hospital discharge diagnosis be used for surveillance of bacteremia? A data quality study of a Danish hospital discharge registry. Infect Control Hosp Epidemiol. 1998;19(3):175-80.

55. Christensen JS, Jensen TG, Kolmos HJ, Pedersen C, Lassen A. Bacteremia with Streptococcus pneumoniae: sepsis and other risk factors for 30-day mortality--a hospital-based cohort study. Eur J Clin Microbiol Infect Dis : official publication of the European Society of Clinical Microbiology. 2012;31(10):2719-25.
56. Bates DW, Pruess KE, Lee TH. How bad are bacteremia and sepsis? Outcomes in a cohort with suspected bacteremia. Arch Intern Med. 1995; 155(6):593-8.

57. Leibovici L, Samra Z, Konigsberger H, Drucker M, Ashkenazi S, Pitlik SD. Longterm survival following bacteremia or fungemia. Jama. 1995;274(10):807-12.

58. Bates DW, Sands K, Miller E, Lanken PN, Hibberd PL, Graman PS, Schwartz JS, Kahn K, Snydman DR, Parsonnet J, et al. Predicting bacteremia in patients with sepsis syndrome. Academic Medical Center consortium Sepsis project working group. J Infect Dis. 1997;176(6):1538-51.

59. Peduzzi P, Shatney C, Sheagren J, Sprung C. Predictors of bacteremia and gram-negative bacteremia in patients with sepsis. The veterans affairs systemic Sepsis cooperative study group. Arch Intern Med. 1992;152(3):529-35.

60. Henriksen DP, Laursen CB, Jensen TG, Hallas J, Pedersen C, Lassen AT. Incidence rate of community-acquired sepsis among hospitalized acute medical patients-a population-based survey. Crit Care Med. 2015;43(1):13-21.

61. Angus DC, Linde-Zwirble WT, Lidicker J, Clermont G, Carcillo J, Pinsky MR. Epidemiology of severe sepsis in the United States: analysis of incidence, outcome, and associated costs of care. Crit Care Med. 2001;29(7):1303-10.

62. Pulia MS, Redwood R, Sharp B. Antimicrobial stewardship in the Management of Sepsis. Emerg Med Clin North Am. 2017;35(1):199-217.

63. Tenney J, Hudson N, Alnifaidy H, Li JTC, Fung KH. Risk factors for aquiring multidrug-resistant organisms in urinary tract infections: a systematic literature review. Saudi Pharm J : SPJ : the official publication of the Saudi Pharmaceutical Society. 2018;26(5):678-84.

64. Aliberti S, Di Pasquale M, Zanaboni AM, Cosentini R, Brambilla AM, Seghezzi S, Tarsia P, Mantero M, Blasi F. Stratifying risk factors for multidrug-resistant pathogens in hospitalized patients coming from the community with pneumonia. Clin Infect Dis. 2012;54(4):470-8.

65. Ruscher C, Pfeifer Y, Layer F, Schaumann R, Levin K, Mielke M. Inguinal skin colonization with multidrug-resistant bacteria among residents of elderly care facilities: frequency, persistence, molecular analysis and clinical impact. Int J Med Microbiol : IJMM. 2014;304(8):1123-34.

66. European Centre for Disease Prevention and Control: Surveillance of antimicrobial resistance in Europe. Annual report of the European Antimicrobial Resistance Surveillance Network (EARS-Net) 2017. Stockholm: ECDC; 2018. (https://ecdc.europa.eu/sites/portal/files/documents/EARS-Netreport-2017-update-jan-2019.pdf).

67. Walter J, Haller S, Blank HP, Eckmanns T, Abu Sin M, Hermes J. Incidence of invasive meticillin-resistant Staphylococcus aureus infections in Germany, 2010 to 2014. Euro surveill : bulletin Europeen sur les maladies transmissibles = European communicable disease bulletin. 2015;20(46). http://dx.doi.org/10.2807/1560-7917.ES.2015.20.46.30067.

68. Prina E, Ranzani OT, Polverino E, Cilloniz C, Ferrer M, Fernandez L, Puig de la Bellacasa J, Menendez R, Mensa J, Torres A. Risk factors associated with potentially antibiotic-resistant pathogens in community-acquired pneumonia. Ann Am Thorac Soc. 2015;12(2):153-60.

\section{Publisher's Note}

Springer Nature remains neutral with regard to jurisdictional claims in published maps and institutional affiliations.

\section{Ready to submit your research? Choose BMC and benefit from:}

- fast, convenient online submission

- thorough peer review by experienced researchers in your field

- rapid publication on acceptance

- support for research data, including large and complex data types

- gold Open Access which fosters wider collaboration and increased citations

- maximum visibility for your research: over $100 \mathrm{M}$ website views per year

At BMC, research is always in progress.

Learn more biomedcentral.com/submissions 